\title{
The Outcome of Endoscopic Surgery on Patients With Sinonasal Inverted Papilloma
}

\author{
Soheila Nikakhlagh ${ }^{1}$; Sana Taiebi ${ }^{1}$; Leila Sistani Karampur ${ }^{1}$; Ghasem Neici ${ }^{2}$; Nader Saki ${ }^{3,{ }^{*}}$ \\ ${ }^{1}$ Hearing and Speech Research Center, Ahvaz Jundishapur University of Medical Sciences, Ahvaz, IR Iran \\ ${ }_{2}^{2}$ Radiology Department, Ahvaz Jundishapur University of Medical Sciences, Ahvaz, IR Iran \\ ${ }^{3}$ Cancer Research Center, Ahvaz Jundishapur University of Medical Sciences, Ahvaz, IR Iran \\ ${ }^{*}$ Corresponding author: Nader Saki, Cancer Research Center, Ahvaz Jundishapur University of Medical Sciences, Ahvaz, IR Iran. Tel: +98-9161187397, Fax: +98-6132921838, \\ E-mail: Ahvaz.ent@gmail.com; Saki-n@ajums.ac.ir
}

Received: June 9, 2015; Accepted: July 12, 2015

\begin{abstract}
Background: Inverted papillomas comprise $0.5 \%$ - $4 \%$ of benign epithelial nasal tumors that arise primarily from the lateral nasal wall. The main characteristic is their tendency to recur and the risk of malignant transformation.

Objectives: The aim of this study was to report on our experience in the treatment of these lesions, especially through the endoscopic approach, and the outcome of the patients.

Patients and Methods: This is a retrospective report describing 38 patients who underwent endoscopic excision or open surgery from March 2001 to March 2012. The patients were studied by consideration of their age, gender, origin of lesion, presenting symptoms, radiological studies, clinical staging, method of treatment, follow up, surgical outcome, tumor recurrence and associated malignancy. The mean follow-up was 47.84 months.

Results: There were 30 males and eight females, and the mean age was 49.76 years ranging from 18 to 78 years. The most common clinical symptoms were unilateral nasal obstruction (94.7\%) and rhinorrhea (36.84\%). Disease recurrence rate was 5.3\%. Endonasal endoscopic resection was performed for 32 patients (84.2\%), combined approaches were used for five patients (13.2\%) and external approach was only done for one patient. The most common involvement location was the maxillary sinus (89\%). The ethmoid sinus and the lateral nasal wall were involved in $68 \%$ and $55.2 \%$ of the cases, respectively. Malignant transformation was observed in two patients (5.3\%).

Conclusions: Long-term follow up of inverted papilloma is necessary for early detection of recurrence and to allow for surgical salvage. Surgeon's experience plays a decisive role in the selection of an appropriate method.
\end{abstract}

Keywords: Papilloma; Inverted; Endoscopic Operative; Open Operative

\section{Background}

Inverted papilloma is a benign sinonasal epithelial neoplasm, which involves the lateral nasal wall. It comprises $0.5 \%$ - $4 \%$ of primary nasal neoplasms, its peak age is during the 5 th or 6 th decade of life and involves males more than females (1). Clinical symptoms depend on the involvement location yet the most common symptom is unilateral and progressive nasal obstruction. Other symptoms usually include unilateral sinonasal discharge yet bilateral sinonasal involvement is reported in $1 \%$ - $9 \%$ of cases (2-4). A pink, polypoid, smooth or lobulated papillary mass seen in the lateral nasal wall above the inferior turbinate in the nasal cavity during examination is suggestive of inverted papilloma that can be differentiated from antrochoanal polyp or other neoplastic lesions (5). Lateral rhinotomy and medial maxillectomy were selected as surgery methods in the past for the treatment of inverted papilloma. Endoscopic surgery is another method, which was proposed during the last decade. Its advantages include avoidance of external incisions, maintenance of the normal physiological function of the mucociliary system and the possibility of regular endoscopic examinations during post-surgery follow-ups (6). Surgeons' views on the priority of surgical methods are different. Several articles have indicated that the recurrence rate of endoscopic surgery is $3 \%-17 \%$, which is less than the recurrence rate of external surgery (7). The highest neoplasm recurrence rate is early recurrence at its primary location, and its most important cause is incomplete removal of the neoplasm. This is why some surgeons prefer the external rhinotomy method over the endoscopic method.

\section{Objectives}

The present study investigated the results and outcome of endoscopic surgery in patients with inverted papilloma at Imam Khomeini and Apadana hospitals of Ahvaz between 2001 and 2012.

\section{Patients and Methods}

This retrospective study was conducted on 38 patients.

Copyright (C) 2015, Ahvaz Jundishapur University of Medical Sciences. This is an open-access article distributed under the terms of the Creative Commons Attribution-NonCommercial 4.0 International License (http://creativecommons.org/licenses/by-nc/4.0/) which permits copy and redistribute the material just in noncommercial usages, provided the original work is properly cited. 
Patients' information from 21st of March 2001 to 19th of March 2012 was extracted along with their pathology results and they were examined for recurrence using sinonasal endoscopy and computer imaging of the sinuses. Their demographic information including age at diagnosis, gender, symptoms, location of neoplasm after diagnosis, and surgery type (endoscopic surgery, lateral rhinotomy and caldwell-Luc surgery) was extracted from their records. They were called and invited for a revisit and endoscopy to check for the recurrence of symptoms. Descriptive statistics and chi-square test were used for providing frequency tables and indices, and comparison of recurrence rates, respectively.

\section{Results}

Among the 38 patients in this study, 30 were male (78.9\%) and eight were female (21.1\%). The male-female ratio was 3.75:1. Patients' age ranged from 18 to 78 years and the mean age was 49.76 years old when the symptoms were diagnosed (Figure 1). The most common symptom was unilat- eral nasal obstruction (94.7\%). The other symptoms included nasal discharge and postnasal drip (36.84\%), headache (13.15\%), nasal bleeding, and loss of the sense of smell (a prevalence of $10.5 \%$ for each) and voice change (2.6\%).

The sinonasal involvement was mostly unilateral. In $42.1 \%, 47.4 \%$ and $10.5 \%$ of cases, involvement was at the right, left and bilateral region, respectively. The most common involvement location was the nasal cavity (89\%) followed by maxillary sinus (68\%), ethmoid sinus (55.2\%), frontal sinus (26.3\%), sphenoid sinus (23.6\%) and nasopharynx (15.87\%). According to the Krouse classification, at diagnosis, $7.89 \%, 39.47 \%, 26.31 \%$ and $26.31 \%$ of patients were in T1, T2, T3 and T4 classes, respectively. Moreover, 32 (84.2\%), five (13.2\%) and one patient underwent endoscopic surgery, endoscopic with caldwell-Luc surgery and lateral rhinotomy surgery, respectively. Among the 38 studied patients, six (15.8\%) underwent surgery after early diagnosis of polyposis during endoscopic examination and imaging, whose side was determined in the pathological diagnosis of sinonasal-inverted papilloma.

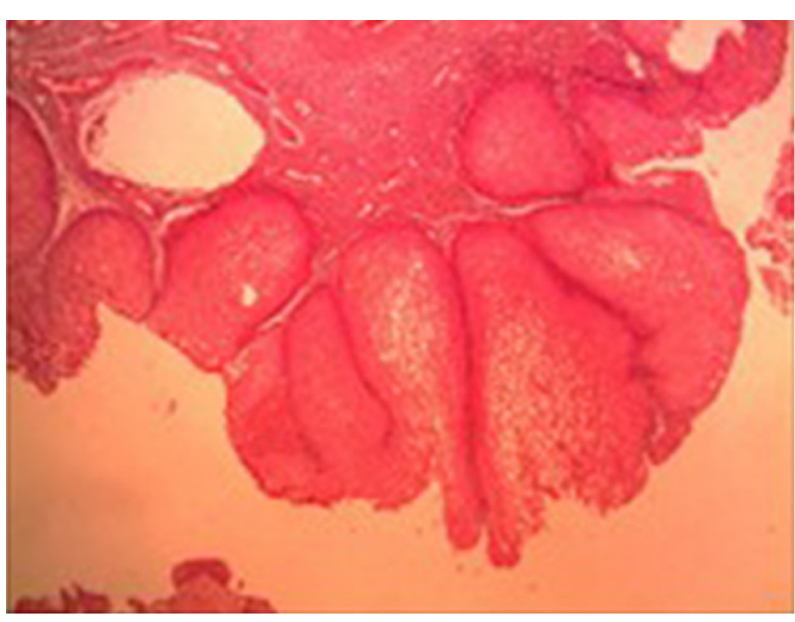

Patholcy of inverted Papiloma

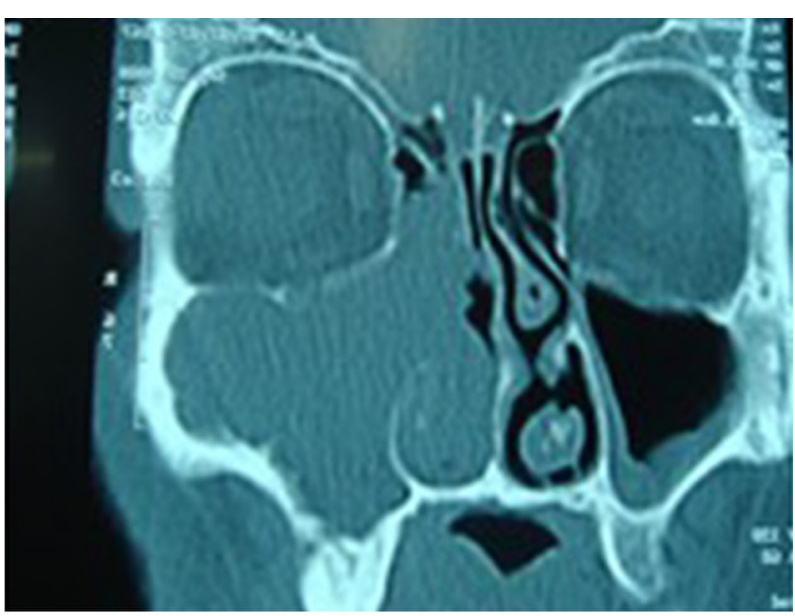

Right side inverted Papilloma

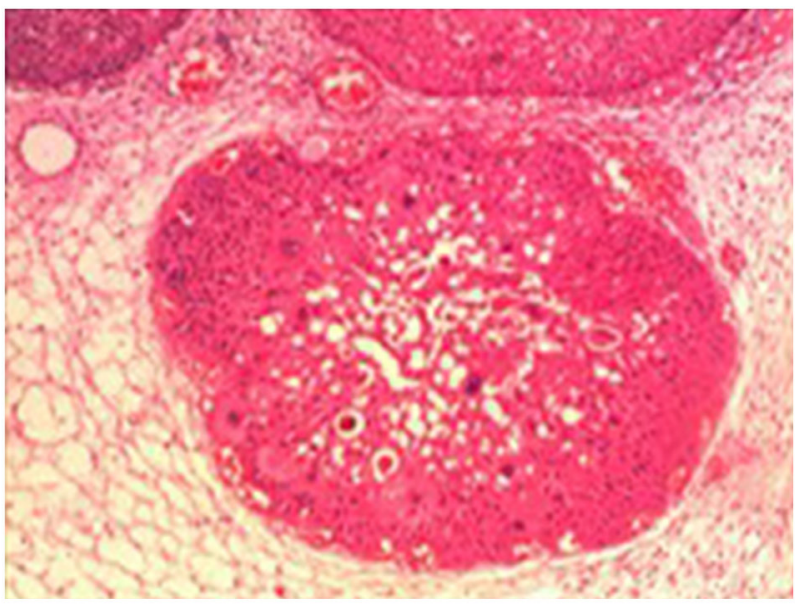

Carcinoma insitu in inverted Papilloma

Figure 1. Distribution of Age 
During follow-up, two patients with squamous carcinoma (5.3\%) were observed, where one case had metachronous and the other synchronous carcinoma, with the diagnosis of inverted papilloma. This patient was a 52-year-old man who had been admitted with nasal obstruction symptoms and nasal mass on the right. His involved sinuses were maxillary, ethmoid, frontal, and sphenoid sinuses. Erosion of lamina papyracea was observed in this patient during an endoscopy surgery. He underwent endoscopy surgery, and severe localized dysplasia (in situ carcinoma) was reported in his pathology (Figure 2). After 36 months of follow up, no evidence of recurrence was observed in this patient. He was diagnosed with synchronous squamous carcinoma along with sinonasal inverted papilloma. The second patient was a 73-year-old man who underwent surgery with nasal obstruction. Bleeding from the right nasal cavity and sinonasal inverted papilloma were reported in his pathology. Eight months after the surgery, symptoms recurred at the same side. After endoscopy surgery and histopathological examination of the sample, the diagnosis of inverted papilloma along with well-differentiated squamous carcinoma was confirmed; the patient then underwent open surgery.

We had recurrence in two cases and isolated frontal sinus involvement was observed in one case. The patient had nasal obstruction and discharge with swelling of the left upper eyelid. Involvement of the frontal sinus with expansion into the orbit and chronic polyposis was observed and the patient underwent surgery with the diagnosis of frontal sinus mucocele with expansion to the left orbit. Periorbital abscess, polyposis, chronic sinusitis and sinonasal inverted papilloma was reported in his pathology. This patient had a history of three surgeries due to polyposis and inverted papilloma was not reported in any of pathology results.

There was no significant relationship between recurrence rate and patients' age and gender based on Fisher's exact statistical test $(P=0.999)$. Recurrence rate was $12.5 \%$ and $16.7 \%$ in females and males, respectively.

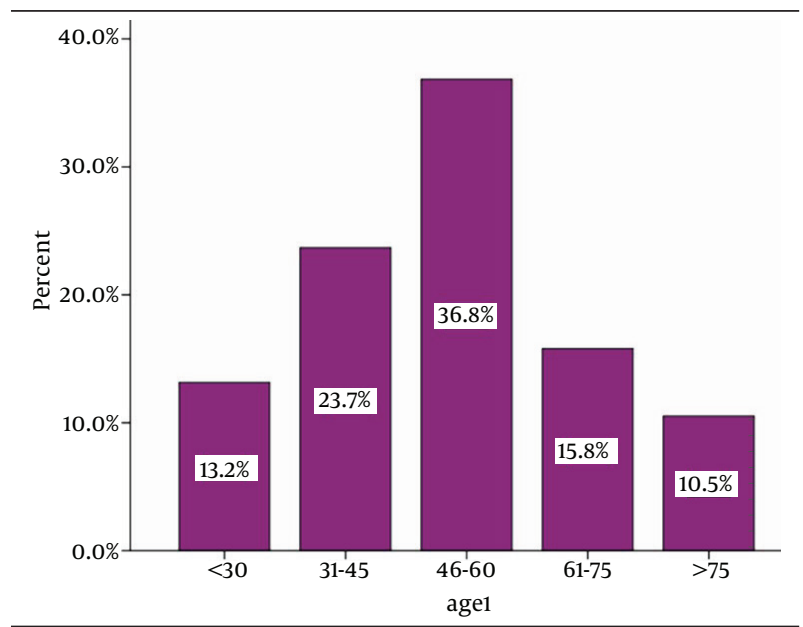

Figure 2. Radiologic and Pathologic Pattern of Inverted Papilloma

\section{Discussion}

Inverted papilloma is a type of sinonasal neoplasm, which can occur at any age. However, the majority of patients are affected during the 5th and 6th decade of their life. This neoplasm is more common in males. Its main characteristic is its tendency to recur and its remodeling and bone destruction ability. Although it is primarily benign, its malignant transformation is well-recognized (8). Clinical symptoms of inverted papilloma are different depending on the extent of involvement of the surrounding structures and the presence of secondary pathology. Unilateral nasal obstruction has been the most common clinical symptom in most studies.

In the past, choice of treatment for patients with sinonasal-inverted papilloma was open surgery, yet nowadays, most articles support the endoscopic method as the selected method for the treatment of these patients (9). The indication of endoscopic surgery is more widespread today and larger neoplasms can be successfully operated with this technique. In the recent years, external surgery methods have been conducted on certain patients in combination with endoscopy (10). In today's world in which surgery and hospitalization duration are two important conditions in surgical planning, endoscopic medial maxillectomy is very useful (11-13). Recurrence rate in external surgery methods for sinonasal-inverted papilloma varies from $0 \%-36 \%$ while this rate is $0 \%-25 \%$ for endoscopic surgery methods $(8,14)$.

It is important to mention that endoscopic surgery of neoplasms depends on the technique and is associated with potential complications. In order to ensure low recurrence rate and avoid complications, endoscopic resection of the neoplasm should be done by skilled and experienced surgeons using endoscopic sinus surgery techniques (11).

Moreover, the role of endoscopy is important in patient follow-up. Careful observation of sinonasal cavity after surgery using endoscopic results is substantial for early diagnosis of the disease and its recurrence $(11,15)$. Moreover, long-term examination is recommended since this complication can recur after years. Recurrent inverted papilloma is associated with the risk of squamous carcinoma and its rate is reported to be $2 \%-53 \%$. With respect to the possibility of secondary malignant transformation, long-term follow-up of patients with nasal endoscopic methods and if required, computerized tomography (CT) scan and Magnetic Resonance Imaging (MRI) is necessary $(15,16)$. Imaging plays an important role in patient evaluation. It determines the extension of the neoplasm and bone erosion or invasion of surrounding structures such as skull base and orbit. These changes can predict associated malignancies and change the type of treatment. Although CT scan can determine the method of inverted papilloma surgery, MRI can reveal neoplasm spread more accurately by differentiating it from the remaining discharged and associated sinusitis (12). 
The majority of this studied patients were males (78.9\%), which is consistent with the majority of studies $(9,12,13)$. Patients' age ranged from 18 to 78 years with the mean age of 49.76 years when symptoms were diagnosed, which is consistent with other studies $(8,9,12)$. The male-female ratio was 3.75:1 in this study. In a study by Sousa et al. this ratio was reported as 1:1 (11), which is inconsistent with the present study. Moreover, in a study by Baradaranfar et al. in Yazd, the male-female ratio was reportedly 11:1 (12), which is inconsistent with the present study. The most common clinical symptom in this study was unilateral nasal obstruction (94.7\%), which is consistent with the majority of studies (9,11-13). The involvement location in this study was mostly unilateral, which was more common on the left. In $10.5 \%$ of patients, the involvement was bilateral, which is inconsistent with the results of the study by Bhandary et al. (13). The present study is consistent with the study by Sousa et al. in which $11.5 \%$ of patients suffered from bilateral sinonasal involvement and the most common involved location was on the right (11). In the study by Baradaranfar, involvement was more common on the left and $12.5 \%$ of patients had bilateral sinonasal involvement (12). The most common involved sinus in this study was the maxillary sinus. This issue has been studied by other researchers as well $(10,11)$. In a study by Diaz Molina, the most common involved sinus was the ethmoid sinus (10). Other involved sinuses in this study included ethmoid, frontal and sphenoid sinuses, in the order of prevalence. This result is rather inconsistent with that of Sousa, in which the least common involved sinus was the frontal sinus (11).

Recurrence rate during this study was 5.3\% (two patients) yet no recurrence was observed during some studies (13). In some studies, recurrence rate was reportedly $7.1 \%(10,12)$. This rate was higher in some studies (about 25\%) (11), which is inconsistent with the present study. Two cases of malignancy associated with inverted papilloma were observed during this study. One case had in situ synchronous carcinoma and the other had metachronous squamous carcinoma, which occurred eight months after the diagnosis of primary inverted papilloma. No evidence of symptoms was observed during the study, 36 and 19 months after the diagnosis of carcinoma. In a study by Bhandary et al. 10.7\% of malignancies were observed with inverted papilloma and all three cases had squamous carcinoma (12), which was consistent with the present study.

In another study by Kim et al. malignancy rate with inverted papilloma was reportedly $7 \%$. There was no significant age difference at diagnosis between these patients and other patients with inverted papilloma. One of these malignancies was transitional carcinoma and the others were squamous carcinoma, most of which were reported to be synchronous (9). In another study by Diaz Molina, $16 \%$ of patients had malignancies along with inverted papilloma. Most of the cases had squamous cell carcinoma and two had transitional carcinoma, most of which were synchronous (10).
Today, endoscopic approaches tend to be the treatment of choice for the majority of inverted papillomas. Close follow-up of the patient for a long period of time is necessary for early detection of recurrence and to allow for surgical salvage. Surgeon's experience is a determining factor when choosing the type of surgery for these cases.

\section{Acknowledgements}

The authors would like to thank the research deputy for their valuable cooperation. This research was the result of a funded medical doctorate thesis (Ref-No. 597).

\section{Authors' Contributions}

Soheila Nikakhlagh, Sana Taiebi, Leila Sistani Karampur, Ghasem Neici did the experimental work. Nader Saki designed, analyzed and wrote the manuscript.

\section{Funding/Support}

Ahvaz Jundishapur University of Medical Sciences.

\section{References}

1. Suh JD, Chiu AG. What are the surveillance recommendations following resection of sinonasal inverted papilloma? Laryngoscope. 2014;124(9):1981-2.

2. Oikawa K, Furuta Y, Nakamaru Y, Oridate N, Fukuda S. Preoperative staging and surgical approaches for sinonasal inverted papilloma. Ann Otol Rhinol Laryngol. 2007;116(9):674-80.

3. Gu FM, Zhang LS. Clinical outcomes of endoscopic and open resection of recurrent sinonasal inverted papilloma. J Craniofac Surg. 2014;25(3):1090-3.

4. Wormald PJ, Ooi E, van Hasselt CA, Nair S. Endoscopic removal of sinonasal inverted papilloma including endoscopic medial maxillectomy. Laryngoscope. 2003;113(5):867-73.

5. Nikakhlagh S, Rahim F, Saki N, Mohammadi H, Maliheh YM. Antrochoanal polyps: report of 94 cases and review the literature. Niger J Med. 2012;21(2):156-9.

6. Busquets JM, Hwang PH. Endoscopic resection of sinonasal inverted papilloma: a meta-analysis. Otolaryngol Head Neck Surg. 2006;134(3):476-82.

7. Han JK, Smith TL, Loehrl T, Toohill RJ, Smith MM. An evolution in the management of sinonasal inverting papilloma. Laryngoscope. 2001;111(8):1395-400.

8. Roh HJ, Procop GW, Batra PS, Citardi MJ, Lanza DC. Inflammation and the pathogenesis of inverted papilloma. Am J Rhinol. 2004;18(2):65-74.

9. Kim K, Kim D, Koo Y, Kim CH, Choi EC, Lee JG, et al. Sinonasal carcinoma associated with inverted papilloma: a report of 16 cases. JCraniomaxillofac Surg. 2012;40(4):e125-9.

10. Diaz Molina JP, Llorente Pendas JL, Rodrigo Tapia JP, Alvarez Marcos C, Obeso Aguera S, Suarez Nieto C. [Inverted sinonasal papillomas. Review of 61 cases]. Acta Otorrinolaringol Esp. 2009;60(6):402-8.

11. Sousa AM, Vicenti AB, Speck Filho J, Cahali MB. Retrospective analysis of 26 cases of inverted nasal papillomas. Braz J Otorhinolaryngol. 2012;78(1):26-30.

12. Baradaranfar MH, Dabirmoghadam P. Trans nasal endoscopic ap proach for sinonasal Inverted papilloma. Med J Islamic Republic Iran. 2004;18(2):127-30.

13. Bhandary S, Singh RK, Sinha AK, Badhu BP, Karki P. Sinonasal inverted papilloma in eastern part of Nepal. Kathmandu Univ Med J (KUMJ). 2006;4(4):431-5.

14. Schlosser RJ, Mason JC, Gross CW. Aggressive endoscopic resection of inverted papilloma: an update. Otolaryngol Head Neck Surg. 2001;125(1):49-53. 


\section{Nikakhlagh S et al.}

15. Sautter NB, Cannady SB, Citardi MJ, Roh HJ, Batra PS. Comparison of open versus endoscopic resection of inverted papilloma. Am J Rhinol. 2007;21(3):320-3.
16. von Buchwald C, Bradley PJ. Risks of malignancy in inverted papilloma of the nose and paranasal sinuses. Curr Opin Otolaryngol Head Neck Surg. 2007;15(2):95-8. 